\title{
A Simplified Frequency Model for Industrial Common- Mode Chocks Used in High-Power Converters
}

\author{
Seyed Fariborz Zarei ${ }^{1{ }^{*}} \cdot$ Saeed Khankalantary ${ }^{2}$
}

\begin{abstract}
This paper proposes a simplified analytical model for electromagnetic interference (EMI) filters used in high-power converters. Highpower converters produce radio frequency conducted noise because they use high-frequency switching in the range of a few $\mathrm{kHz}$ to tens of $\mathrm{kHz}$. The noise propagates into the power grid, which disturbs the functionality of the radio frequency apparatuses. Well-known standards, such as CISPR, provide the measurement and assessment methodologies for these devices. Moreover, the emission level of the noise is restricted at the source side. Using EMI filters is the most effective approach for dealing with this issue. However, due to the nonlinear nature of the common-mode $(\mathrm{CM})$ cores, the modeling of the cores is a complicated task, which makes the selection and design of the filters less than optimal. In this paper, an analytical modeling of the CM filters is provided to suit the nonlinear frequency-dependent behavior of the CM cores. The simplicity of the proposed model makes it a very suitable choice for inclusion in the design procedures, which results in a more accurate and optimum filter selection among the many available commercial industrial filters. To validate the proposed model, the frequency-dependent model obtained is verified by experimental tests with a commercial CM choke. According to the results, the proposed model accurately describes the actual EMI filter behavior.
\end{abstract}

Key Words: Common-Mode Chock, Electromagnetic Interference (EMI), EMI Filters, Power Electronic Converters.

\section{INTRODUCTION}

High-frequency conducted emissions produced by power electronic converters leak to neighboring electrical systems and equipment, causing electromagnetic interference (EMI) $[1,2]$. Conducted emissions degrade or even disturb the performance of the electrical apparatus, and therefore, the emission levels are restricted by defining different limits. The measurement techniques and assessment criteria are developed according to international standards, such as IEC and CISPR [3, 4]. To adhere to the standard levels, limiting noise on the source side is the best and most cost-effective remedy [5]. EMI filters are wellknown for suppressing the noise, as they provide a high imped- ance path against the conducted noise [6]. In this regard, EMI filter design is a critical step in the filtering process, and the modeling of common-mode (CM) filters for high-power converter applications is discussed in this paper.

In CM filters, the net flux of CM cores only contains zerosequence noises, which are mainly non-fundamental highfrequency components [7]. Nonlinear characteristics versus the operating frequencies of $\mathrm{CM}$ cores make the analysis of such filters difficult [8]. In this regard, modeling the filters in the whole operating range is a challenge. Two types of designers take on different tasks to with this modeling problem:

(i) Filter designers focus on the initial design of CM filters, considering the proper magnetic core material and shape selec-

Manuscript received March 9, 2020 ; Revised June 3, 2020 ; Accepted August 31, 2020. (ID No. 2020309-026J)

${ }^{1}$ Department of Electrical and Computer Engineering, Qom University of Technology, Qom, Iran.

${ }^{2}$ Department of Electrical and Computer Engineering, Isfahan University of Technology, Isfahan, Iran.

"Corresponding Author: Seyed Fariborz Zarei (e-mail: zarei@qut.ac.ir)

This is an Open-Access article distributed under the terms of the Creative Commons Attribution Non-Commercial License (http://creativecommons.org/licenses/by-nc/4.0) which permits unrestricted non-commercial use, distribution, and reproduction in any medium, provided the original work is properly cited.

(c) Copyright The Korean Institute of Electromagnetic Engineering and Science. All Rights Reserved. 
tion, calculating the number of turns, and employing proper winding schemes. Achieving sufficient insertion loss characteristics for the CM filter is the goal of such design procedures.

(ii) Circuit designers are intended to select the best filter among numerous commercially available filters for their specific applications.

The filter designers try to provide the best filters with the proper characteristics for the whole radio frequency range. On the other hand, the circuit designers try to select the most appropriate filter among the many available industrial filters for the specific circuit. From both points of view, filter modeling is necessary, and there are two separate efforts for modeling: proposing the proper topology for the model, and proposing the methodology for extracting the parameters of the topology. In the following, the existing research is presented.

To model CM filters, different approaches are provided in the literature. Insertion loss measurement in a $50 \Omega / 50 \Omega$ system is the basic method. However, the insertion loss of a filter in the operating condition is not the same as in a measured condition [9]. To solve the issue, different approaches, such as mixed-mode $S$-parameters and modal models, have been proposed $[10,11]$, which adds even more complexity to the modeling process [12]. Consequently, impedance measurement-based methods were developed and are now the most frequently used techniques for modeling passive filters [13]. In this respect, a lumped-element high-frequency model for $\mathrm{CM}$ chokes is utilized in $[13,14]$. To extract the parameters of the model, heuristic methods [15], rational function approximation $[8,16]$, iterative rational function [12], and fitting methods [17] are employed. Furthermore, transfer function-based modeling is also employed, in which the numerator and denominator coefficients are obtained by implementing the experimental measurements into the available system identification toolboxes [18]. To summarize these previous studies, the topology of the highfrequency model is either complex or chosen heuristically, which requires significant experience and testing. Furthermore, although the estimated models are accurate, the circuit diagram of the filter is complex. These concerns make the existing schemes complicated for modeling, especially from the circuit designer's perspective, for which a simple but accurate modeling approach is needed.

The parasitic element extraction of the CM filters is discussed in another study; it includes a variety of approaches, such as solving a $3 \mathrm{D}$ electromagnetic model of CM filters [19, 20], taking experimental impedance measurements and fitting the obtained data into the physical or behavioral circuits [16, 17], modal analysis of the CM circuit model [18], and obtaining transmission coefficients using a four-point network concept [21]. The impact of parasitic elements dominates above the
$\mathrm{MHz}$ frequency band, which is beyond the frequency range of this study. In other words, from the circuit designer's perspective, the aggregated parasitic capacitances of the converter and loads cause resonances in the $\mathrm{CM}$ currents in frequencies below $\mathrm{MHz}$ in high-power converter applications $[22,23]$. The circuit designer's task is then to keep these noise levels below the standard limit values. For this reason, this range of frequency $(150 \mathrm{kHz}$ to a few $\mathrm{MHz})$ is the focus of this paper, in which the CM magnetic core characteristics play a more prominent role compared to the parasitic capacitances.

This paper proposes an analytical model for CM filters for high-power converters that can be obtained simply through the measurement results from manufacturers' datasheets. In the proposed method, the physical frequency-dependent behavior of the CM magnetic cores as informed by the complex permeability concept is adopted as a base for modeling and topology extraction. Accordingly, a simple frequency-dependent series connected resistance-inductance circuit model is developed. The model coefficients and equivalent circuit parameters are then extracted using the measurement data available from the $\mathrm{da}-$ tasheets of commercially available industrial CM filters. Simplicity and accuracy are the key features of the proposed model, which is useful for circuit designers, as it has many available choices for selection. Using this model, the designer can efficiently and simply analyze the different commercially available $\mathrm{CM}$ filters in the circuit studied.

The rest of the paper is organized as follows. Section II describes the function of the CM filters, focusing on their noisefiltering capability. Section III presents the proposed formulation and EMI filter modeling. In the next step, in Section IV, the proposed methodology for obtaining the model parameters is presented. In this section, the parameters are obtained for a commercially available CM filter using the experimental measurement results. Finally, Section V concludes the paper.

\section{EMI FILTERS FOR SUPPRESSING COMMON-MODE NOISE}

EMI filters for CM conducted emissions effectively suppress noise using the two well-known configurations shown in Fig. 1, namely, bifilar and sectional configurations [24, 25]. In both configurations, the $\mathrm{CM}$ current results in a net flux in the core, and the core then behaves as an inductor against the $\mathrm{CM}$ current. This means that the CM filter appears as a high-impedance path when the $\mathrm{CM}$ current flows, resulting in a reduction of the CM currents. Despite the CM currents, the flux of DM currents cancel each other in the CM core, resulting in a near-zero net flux. The CM filter thus appears as a negligible impedance against DM currents. As a result, the net flux seen by the $\mathrm{CM}$ core is produced by $\mathrm{CM}$ currents, which have domi- 


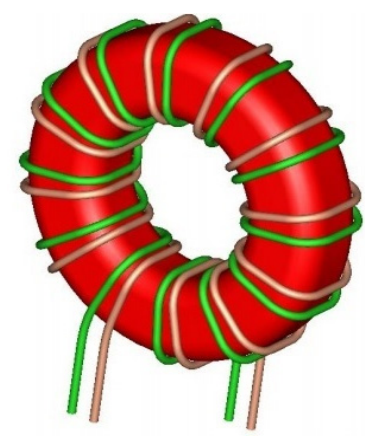

(a)

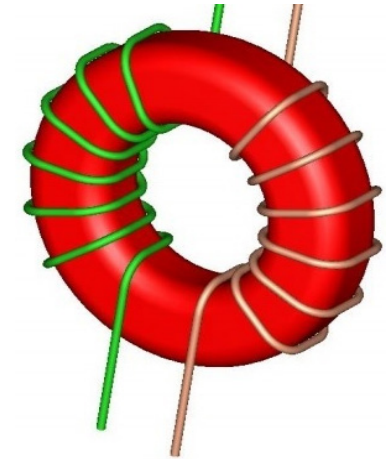

(b)
Fig. 1. Common-mode filter: (a) bifilar configuration and (b) sectional configuration. Adapted from Raeker [25].

nantly high-frequency content. On the other hand, the CM cores show a nonlinear frequency-dependent characteristic, resulting in difficulties in the analysis and modeling of the filters. To deal with this issue, the proposed methodology and modeling are presented in the next section.

\section{PROPOSED ANALYTICAL FORMULATION AND}

\section{FILTER MODELLING}

To model the CM EMI filters, understanding the behavior of the CM cores is the key factor. Generally, in electromagnetism, the magnetic field strength $(\mathrm{H})$ and magnetic flux density (B) are related to each other depending on the given operating medium, as shown in (1). This relationship specifies the organization of magnetic dipoles in the medium, including dipole migration and magnetic dipole reorientation [26]. The ratio of (1) is defined as permeability, which is a scalar if the medium is isotropic or a second rank tensor for an anisotropic medium [27].

$$
\mu=\frac{B}{H}
$$

Generally, permeability is not a constant value since it changes depending on the frequency of the magnetic field, the humidity, and temperature of the medium, and etc. Since the EMI cores used in power electronic applications observe different high-frequency currents, the frequency behavior is the key concern, which is covered in this paper.

Complex permeability is a well-defined concept for analyzing the behavior of the core under high-frequency magnetic fields. According to this concept, (i) at low frequencies, the permeability is a scalar quantity, and (ii) at high frequencies, $\mathrm{B}$ and $\mathrm{H}$ are not proportional by a simple scalar value, and these quantities have a lag time to each other. At high frequencies, $\mathrm{B}$ and $\mathrm{H}$ can be expressed by phasors, as presented in Eq. (2) [28].

$$
H=H_{0} e^{j \omega t} ; \quad B=B_{0} e^{j(\omega t-\delta)}
$$

where $\delta$ is the phase difference between $\mathrm{B}$ and $\mathrm{H}$.

Since permeability is defined as the ratio of the magnetic flux density to the magnetic field, Eq. (3) shows the permeability.

$$
\mu=\frac{B_{0}}{H_{0}} e^{-j \delta}
$$

Thus, the permeability is a complex quantity. Eq. (4) represents the Cartesian form of (3).

$$
\mu=\frac{B_{0}}{H_{0}} \cos (\delta)-j \frac{B_{0}}{H_{0}} \sin (\delta)
$$

Defining real $\left(\mu^{\prime}\right)$ and imaginary $\left(\mu^{\prime \prime}\right)$ permeabilities, the complex permeability of Eq. (4) is reformed to Eq. (5).

$$
\mu=\mu^{\prime}-j \mu^{\prime \prime}
$$

According to Eqs. (4)-(5), the loss tangent is defined as the ratio of the imaginary to the real part of the complex permeability, which yields an index showing how much power is lost in the material versus how much is stored.

$$
\tan (\delta)=\frac{\mu^{\prime \prime}}{\mu^{\prime}}
$$

The frequency-dependent $\mu^{\prime}$ and $\mu^{\prime \prime}$ may be physically related to each other by a physical relation, such as the KramersKronig relation for non-passive materials [29]. A more accurate expression is provided by [30], which is accurate even for passive materials. However, the physics-based modeling of a CM filter or its components necessitates extensive electromagnetic analysis, which makes it very complicated and limits the applicability to specific applications [12, 31, 32]. For this reason, measurementbased modeling approaches are widely used for EMI filters in the field of power electronics. These modeling methods are more general, direct, and accurate for describing the behavior and characteristics of the CM filters [12], and they are also used in this paper. Fig. 2 shows the permeability versus frequency characteristics of ferrite and nanocrystalline $\mathrm{CM}$ cores, which are the most well-defined and widely-used magnetic core materials in CM noise filtering in power electronics [33]. As can be seen from Fig. 2, there is a cut-off frequency for both cores in which the permeability curve starts to decrease from the initial value. In this frequency, $\mu^{\prime}$ start to decrease, and $\mu^{\prime \prime}$ would be the dominant component. According to the conducted emission regulations, the EMI studies are performed in the frequency range of $150 \mathrm{kHz}$ to $30 \mathrm{MHz}$. However, the frequency range of $10 \mathrm{kHz}$ to $1 \mathrm{MHz}$ is considered in this paper, where magnetic core characteristics play a dominant role rather than the parasitic capacitance of the inductor. In this respect, it should be noted that the resonance in the common-mode currents with large peak values occurs in the specified frequency range in high- 


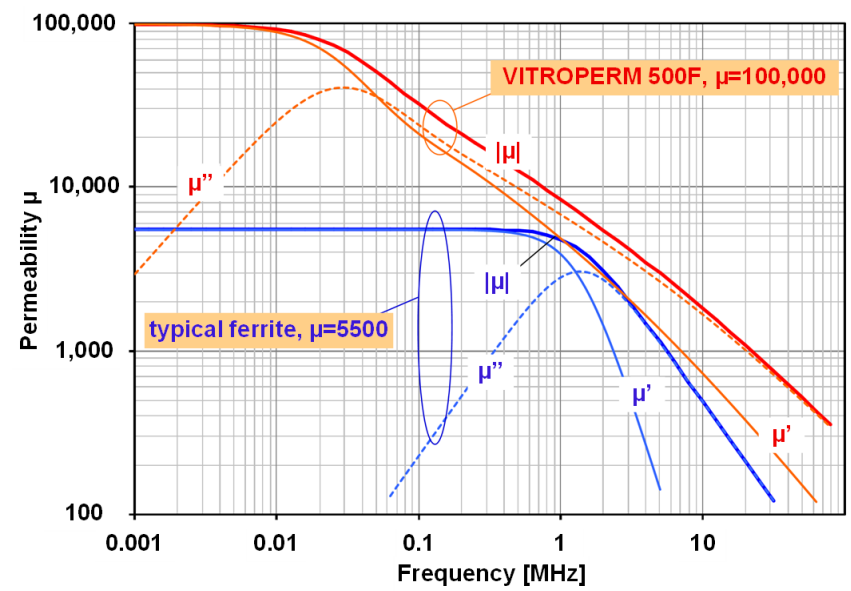

Fig. 2. The permeability versus frequency characteristics of ferrite and nanocrystalline $\mathrm{CM}$ cores. Adapted from VACUUMSCHMELZE [33].

power converter applications, as can be seen in [22, 23]. Modeling of the filters in this range is needed for the circuit designer to calculate and limit the peak of common-mode currents to the standard levels. Accordingly, $\mu^{\prime}$ and $\mu^{\prime \prime}$ are modeled in the specified frequency band according to the experimental results obtained in Fig. 2 for ferrite and nanocrystalline CM cores.

To find the CM core model, the general impedance form of Eq. (7) can be used for the given frequency of $\omega$.

$$
Z=j \mu \frac{N^{2} A}{l} \omega
$$

where, $N, A, \omega$, and $l$ specify the number of turns, crosssection area, angular frequency, and length of the flux path.

Substituting Eq. (5) into Eq. (7) results in (8), which shows the $\mathrm{CM}$ core behavior versus the frequency.

$$
Z=\mu^{\prime \prime} \frac{N^{2} A}{l} \omega+j \mu^{\prime} \frac{N^{2} A}{l} \omega
$$

As can be seen from Eq. (8), the impedance has resistive and inductive parts in which $\mu^{\prime \prime}$ and $\mu^{\prime}$ are frequency-dependent components, as shown in Fig. 2. Below the cut-off frequency, $\mu^{\prime}$ is the dominant component, and $\mu^{\prime \prime}$ can be ignored due to its negligible value compared with $\mu^{\prime}$. However, in higher frequencies, the $\mu^{\prime \prime}$ component becomes larger, introducing a resistive part. Then, the impedance can be represented by Eq. (9).

$$
\left\{\begin{array}{cc}
\overbrace{1}=j \mu_{1}^{\prime} \frac{N^{2} A}{l} \omega & f \leq f_{c u t} \\
Z_{2}=\underbrace{L_{21}^{\prime \prime} \frac{N^{2} A}{l} \omega}_{r_{c 2}}+j \underbrace{\mu_{2}^{\prime} \frac{N^{2} A}{l}}_{L_{c 2}} \omega & f>f_{c u t}
\end{array}\right.
$$

In this equation, $\mu_{1}^{\prime}$ is the constant value, since $f \leq f_{\text {cut }}$.
On the other hand, $\mu_{2}^{\prime \prime}$ and $\mu_{2}^{\prime}$ are frequency-dependent, with a linear relationship in the logarithmic axis. With this equation in mind, the CM core can be modeled by the series connection of a variable (versus frequency) resistance and inductance, as given in Eq. (10).

Eq. (10) shows the complete model of a CM choke. In the next section, the parameter values of the proposed model are obtained using the experimental test results of a commercially available CM filter.

$$
\begin{aligned}
& \left\{\begin{array}{c}
r_{c 1}=0 \\
x_{c 1}=\mu_{1}^{\prime} \frac{N^{2} A}{l} \omega
\end{array} f \leq f_{c u t}\right. \\
& \left\{\begin{array}{l}
r_{c 2}=\mu_{2}^{\prime \prime} \frac{N^{2} A}{l} \omega \\
x_{c 2}=\mu_{2}^{\prime} \frac{N^{2} A}{l} \omega
\end{array} f>f_{c u t}\right.
\end{aligned}
$$

\section{MODEL PARAMETER ESTIMATION USING EXPERIMENTAL MEASUREMENTS}

In this section, the measurement test results of a commercially available CM filter are used for modeling purposes, and accordingly, its related circuit parameters are obtained. The frequency range of $150 \mathrm{kHz}-1 \mathrm{MHz}$ is selected for this study, where the CM core non-linear characteristic plays the dominant role. The experimental impedance measurement versus frequency of the filter is shown in Fig. 3. The following important observations can be derived from the measured impedance.

- As expected from the analysis of the previous section, there is a cut-off frequency for this filter at $90 \mathrm{kHz}\left(f_{\text {cut }}=90 \mathrm{kHz}\right)$.

- Below this frequency $f_{c u t}$, the impedance characteristic is mainly inductive and the resistive part is negligible, as expected.

- Above the cut-off frequency, the resistive part becomes dominant and the slope of the curve decreases noticeably, as expected.

To find the equivalent circuit of the filter, the impedance characteristic of Fig. 3 is approximated by using linear equations in the logarithmic axis as follows.

$$
\begin{cases}\log _{10}\left(\left|Z_{1}\right|\right)=A_{1} \times \log _{10}(f)+B_{1} & f \leq f_{\text {cut }} \\ \log _{10}\left(\left|Z_{2}\right|\right)=A_{2} \times \log _{10}(f)+B_{2} & f>f_{\text {cut }}\end{cases}
$$

Taking the logarithm from the two sides of Eq. (9), the equivalent of Eq. (11) is found as given in Eq. (12), where the values are functions of the frequency.

$$
\begin{cases}\log _{10}\left(\left|Z_{1}\right|\right)=\log _{10}\left(2 \pi \mu_{1}^{\prime} \frac{N^{2} A}{l}\right)+\log _{10}(f) & f \leq f_{\text {cut }} \\ \log _{10}\left(\left|Z_{2}\right|\right)=\log _{10}\left(2 \pi \frac{N^{2} A}{l} f \times \sqrt{{\mu_{2}^{\prime \prime 2}+\mu_{2}^{\prime 2}}^{2}}\right) & f>f_{\text {cut }}\end{cases}
$$




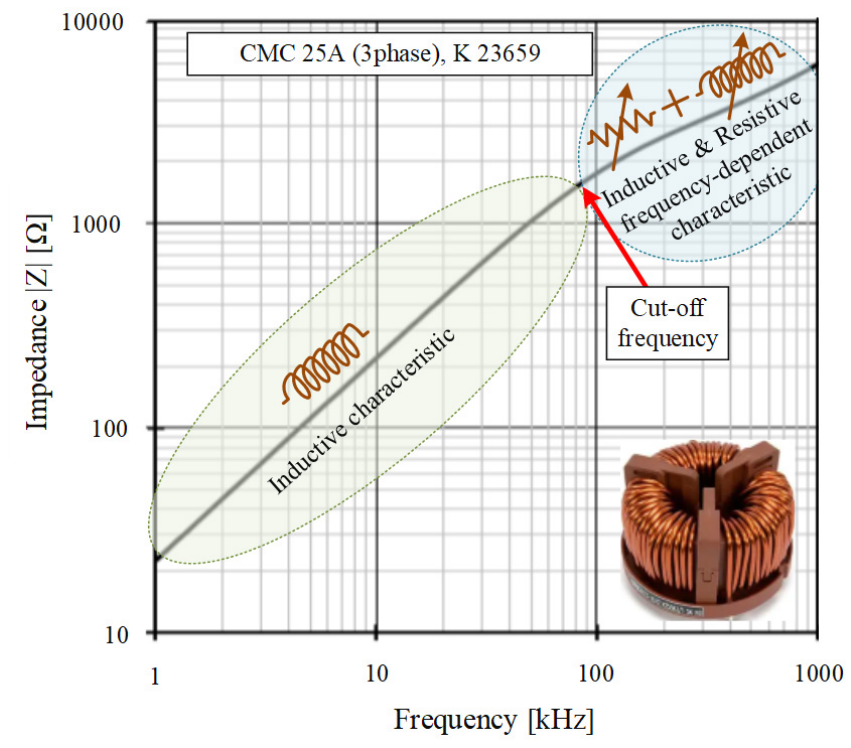

Fig. 3. CM filter impedance versus frequency for a commercially available CM filter obtained by experimental measurements. Adapted from VACUUMSCHMELZE [34].

Here, $\mu_{1}^{\prime}$ is a constant value, and $\mu_{2}^{\prime}$ and $\mu_{2}^{\prime \prime}$ are frequency-dependent components (see Fig. 2). $\mu_{1}^{\prime} \frac{N^{2} A}{l}$ represents the inductance for the frequencies below $f_{\text {cut }}$. To further analyze the equations of Eq. (12), the different parts are separated as follows.

$$
\begin{gathered}
\log _{10}\left(\left|Z_{1}\right|\right)=\log _{10}(f)+B_{1} \\
\log _{10}\left(\left|Z_{2}\right|\right)=\log _{10}\left(2 \pi \frac{N^{2} A}{l}\right)+\log _{10}\left(\sqrt{\mu_{2}^{\prime \prime 2}+\mu_{2}^{\prime 2}}\right) \\
+\log _{10}(f)
\end{gathered}
$$

Here, $B_{1}$ is the unknown but constant value. Moreover, $\mu_{2}^{\prime}$ and $\mu_{2}^{\prime \prime}$ are both functions of frequency in the logarithmic axis, which can be approximated by the first-order function (see Fig. 2). Eq. (14) can then be simplified to Eq. (15), in which $A_{2}$ and $B_{2}$ are unknown values that can be calculated from the experimental results of Fig. 3.

$$
\log _{10}\left(\left|Z_{2}\right|\right)=A_{2} \times \log _{10}(f)+B_{2}
$$

From the above results, it is clear that the impedances of Eq. (11) are in the forms of Eqs. (13) and (15), verifying the validity and accuracy of the modeling presented in the previous section. Finding the values of the model parameters is the next step, which is outlined in the two following subsections.

\section{CM Choke Impedance Interpolation}

In the first step, the impedance function versus frequency is obtained using the measured impedance of the CM choke (see Fig. 3). In this respect, the least square method is applied to Eqs.
(13) and (15) (or equivalently, (11)), and the following shows the resulting function.

$$
\begin{cases}\log _{10}\left(\left|Z_{1}\right|\right)=0.962 \times \log _{10}(f)-1.5 & f \leq f_{\text {cut }} \\ \log _{10}\left(\left|Z_{2}\right|\right)=0.516 \times \log _{10}(f)+2.23 & f>f_{\text {cut }}\end{cases}
$$

The functions obtained in Eq. (16) satisfy the following criteria. As can be seen from the numbers, the proposed model is perfectly fitted to the experimental results, again, verifying the accurate modeling of the CM filters (Table 1).

\section{Model Parameter Estimation}

In this sub-section, the circuit parameters of Eq. (10) are obtained using the equivalent impedances of Eq. (16). As the parameters are functions of $\mu_{2}^{\prime}$ and $\mu_{2}^{\prime \prime}$ and both are unknown variables, it is difficult to obtain a closed formulation for finding these variables. For this reason, a heuristic method should be employed to find the best values according to the measured experimental data. Knowing that $\mu_{2}^{\prime}$ and $\mu_{2}^{\prime \prime}$ are both functions of frequency (see Eq. (17)), substituting Eq. (17) into Eq. (10) results in Eq. (18). For the sake of brevity, only $f>f_{\text {cut }}$ is elaborated in the following. For $f \leq f_{\text {cut }}$, the EMI filter is dominantly inductive (see (10)), and the inductance value can be obtained simply through the experimental tests provided in the datasheets (normally at $10 \mathrm{kHz}$ ).

$$
\begin{gathered}
\log \left(\mu_{2}^{\prime}\right)=a_{2}^{\prime} \times \log (f)+b_{2}^{\prime} \\
\log \left(\mu_{2}^{\prime \prime}\right)=a_{2}^{\prime \prime} \times \log (f)+b_{2}^{\prime \prime} \\
r_{c 2}=10^{\left(a_{2}^{\prime \prime} \times \log (f)+b_{2}^{\prime \prime}\right)} \times \frac{N^{2} A}{l} \omega \\
L_{c 2}=10^{\left(a_{2}^{\prime} \times \log (f)+b_{2}^{\prime}\right)} \times \frac{N^{2} A}{l} \quad f>f_{c u t}
\end{gathered}
$$

Defining $a_{2}^{\prime}, b_{2}^{\prime}, a_{2}^{\prime \prime}, b_{2}^{\prime \prime}$, and $\frac{N^{2} A}{l}$ as unknown variables, and remembering the equivalent impedance from the experimental results of Fig. 3 (see Eq. (16)), a genetic algorithm (GA) is employed to find the variables. For the sake of brevity, GA is not further elaborated. The GA settings are selected as follows.

- $\mathrm{nPop}=700$ (number of populations)

- MaxIt $=700$ (maximum number of iterations)

- FunTolerance $=1 \mathrm{e}-7$

Table 1. Criteria for evaluating goodness of fit according to Eq. (16)

\begin{tabular}{lcc}
\hline & $f \leq f_{\text {cut }}$ & $f>f_{\text {cut }}$ \\
\hline SSE & 0.001417 & 0.000483 \\
$\mathrm{R}^{2}$ & 0.9996 & 0.9992 \\
Adjusted $\mathrm{R}^{2}$ & 0.9995 & 0.9991 \\
RMSE & 0.01044 & 0.006344
\end{tabular}

$\mathrm{SSE}=$ sum of the squared estimate of errors, RMSE=root mean square error. 
Running the genetic algorithm, the unknown variables are found, which are:

$$
\begin{array}{cc}
a_{2}^{\prime}=-0.4843 & b_{2}^{\prime}=4.1347 \quad a_{2}^{\prime \prime}=-34.0253 \\
b_{2}^{\prime \prime}=206.6083 & N^{2} A / l=0.0126
\end{array}
$$

Substituting the obtained values into Eq. (18), the equivalent impedance of the filter is achieved. Fig. 4 shows the measured and the obtained (using the proposed method) impedances of the CM filter versus frequency. As can be seen from this figure, the obtained impedance perfectly matches the measured impedance, verifying the correctness and accuracy of the modeling of the $\mathrm{CM}$ filter.

It is worth noting that both Eqs. (15) and (18) describe the impedance characteristics of the CM chock. However, Eq. (15) only gives the absolute value of the impedance, which is taken from the experiment results. This equation would not be sufficient for circuit analysis, since the resistive-inductive parts of the impedance are not specified. Eq. (18) provides resistive-inductive components of the impedance by combining the measured impedance characteristics of Eq. (15) with the frequency behavior of magnetic materials given in Fig. 2. This means that although Eq. (15) gives the impedance characteristics of CM chocks, Eq. (18) is necessary for circuit designers to obtain an accurate model of the CM chocks that can be employed in their analyses.

\section{CONCLUSION}

This paper has proposed an analytical modeling for EMI filters in high-power converter applications, in which the frequency-dependent behavior of the EMI cores plays the key role. In this respect, the EMI cores' characteristics in response to cur-

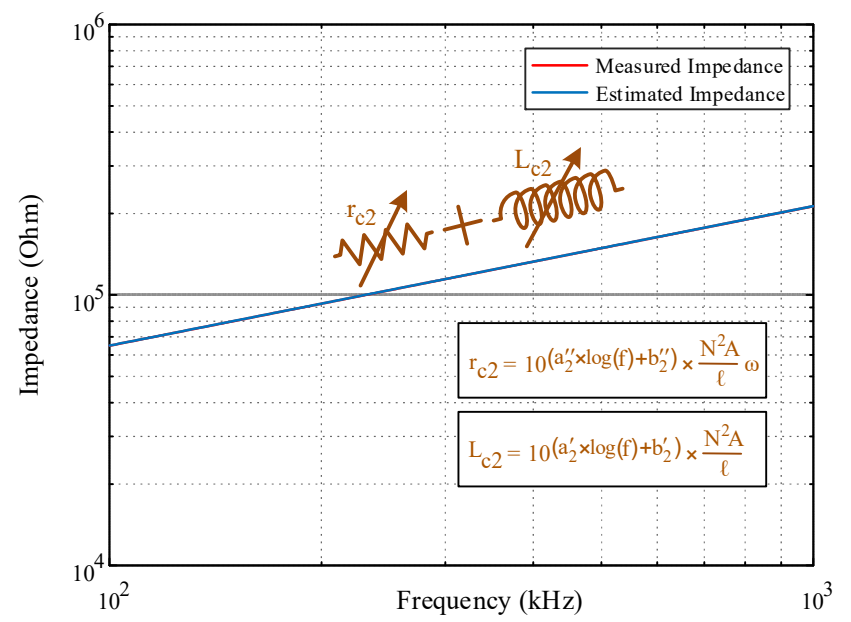

Fig. 4. Impedance of a commercial CM filter obtained by experimental measurements and impedance obtained using the proposed model, both versus frequency $(100 \mathrm{kHz}-1$ $\mathrm{MHz})$. rents with different frequencies have been investigated. It was shown that the EMI cores have nonlinear characteristics versus frequency, which complicates the modeling of the EMI filters. Taking into consideration the nonlinear permeability versus frequency curve of the cores, an analytical formulation of $\mathrm{CM}$ filter impedance has been proposed that offers a simple topology for CM filters. According to this formulation, a model was obtained for a commercially available EMI filter; using the impedance measured by experiments, the proposed model parameters have been calculated. We conclude that the proposed model perfectly matches the measured data, verifying the correct and accurate modeling of the $\mathrm{CM}$ filter. The proposed model can be used in EMI studies and EMI filter design procedures, which increases their accuracy and reduces their cost by selecting proper and optimum EMI filters.

\section{REFERENCES}

[1] B. Narayanasamy and F. Luo, "A survey of active EMI filters for conducted EMI noise reduction in power electronic converters," IEEE Transactions on Electromagnetic Compatibility, vol. 61, no 6, pp. 2040-2049, 2019.

[2] E. M. Kim, M. H. Jeon, D. I. Kim, and Y. S. Ahn, "Development of EMC filter in the high powered breaker," Journal of the Korea Electromagnetic Engineering Society, vol. 9, no. 2, pp. 53-60, 2009.

[3] S. Braun, T. Donauer, and P. Russer, "A real-time timedomain EMI measurement system for full-compliance measurements according to CISPR 16-1-1," IEEE Transactions on Electromagnetic Compatibility, vol. 50, no. 2, pp. 259-267, 2008.

[4] C. Hoffmann, H. H. Slim, and P. Russer, "System strategies for time-domain emission measurements above 1 GHz," Journal of Electromagnetic Engineering and Science, vol. 11, no. 4, pp. 304-310, 2011.

[5] R. Smolenski, Conducted Electromagnetic Interference (EMI) in Smart Grids. London, UK: Springer-Verlag, 2012.

[6] S. Jiang, Y. Liu, W. Liang, J. Peng, and H. Jiang, "Active EMI filter design with a modified LCL-LC filter for single-phase grid-connected inverter in vehicle-to-grid application," IEEE Transactions on Vehicular Technology, vol. 68, no. 11, pp. 10639-10650, 2019.

[7] D. H. Bae, D. I. Kim, and J. M. Song, "Development of improved EMC filter for EFT in power supply," Journal of Electromagnetic Engineering and Science, vol. 1, no. 1, pp. 100-104, 2001.

[8] W. Tan, C. Cuellar, X. Margueron, and N. Idir, "Automatic identification of magnetic component equivalent circuits using impedance measurements," in Proceedings of 2011 IEEE International Instrumentation and Measurement Tech- 
nology Conference, Hangzhou, China, 2011, pp. 1-6.

[9] P. Kong, Y. Jiang, and F. C. Lee, "Common mode EMI noise characteristics of low-power AC-DC converters," IEEE Transactions on Power Electronics, vol. 27, no. 2, pp. 731-738, 2011.

[10] K. Kostov and J. Kyyra, "Insertion loss in terms of fourport network parameters," IET Science, Measurement E Technology, vol. 3, no. 3, pp. 208-216, 2009.

[11] A. M. Sanchez, A. Perez, J. R. Regue, M. Ribo, P. Rodriguez-Cepeda, and F. J. Pajares, "A modal model of common-mode chokes for conducted interference prediction," IEEE Transactions on Electromagnetic Compatibility, vol. 52, no. 3, pp. 749-752, 2010.

[12] W. Tan, C. Cuellar, X. Margueron, and N. Idir, "A high frequency equivalent circuit and parameter extraction procedure for common mode choke in the EMI filter," IEEE Transactions on Power Electronics, vol. 28, no. 3, pp. 11571166, 2012.

[13] J. L. Kotny, X. Margueron, and N. Idir, "High-frequency model of the coupled inductors used in EMI filters," IEEE Transactions on Power Electronics, vol. 27, no. 6, pp. 28052812, 2011.

[14] J. L. Kotny, X. Margueron, and N. Idir, "High frequency modeling method of EMI filters," in Proceedings of 2009 IEEE Energy Conversion Congress and Exposition, San Jose, CA, 2009, pp. 1671-1678.

[15] S. Skibin and I. Stevanovic, "Behavioral circuit modeling of chokes with multi-resonances using genetic algorithm," in Proceedings of 2011 IEEE International Symposium on Electromagnetic Compatibility, Long Beach, CA, 2011, pp. 454-458.

[16] D. H. Liu and J. G. Jiang, "High frequency characteristic analysis of EMI filter in switch mode power supply (SMPS)," in Proceedings of 2002 IEEE 33rd Annual IEEE Power Electronics Specialists Conference (Cat. No. $02 \mathrm{CH}$ 37289), Cairns, Australia, 2002, pp. 2039-2043.

[17] K. Nomura, N. Kikuchi, Y. Watanabe, S. Inoue, and Y. Hattori, "Novel spice model for common mode choke including complex permeability," in Proceedings of 2016 IEEE Applied Power Electronics Conference and Exposition (APEC), Long Beach, CA, 2016, pp. 3146-3152.

[18] M. Illia, L. Koleff, and G. Griepentrog, "Non-ideal model of the common mode choke for EMI filters," in Proceedings of 2017 IEEE Applied Power Electronics Conference and Exposition (APEC), Tampa, FL, 2017, pp. 938-944.

[19] The ECPE WBG Working Group, "ECPE position paper on next generation power electronics based on wide bandgap devices," 2016; https://www.ecpe.org/research/road maps-strategy-papers/strategy-papers/.

[20] M. L. Heldwein, L. Dalessandro, and J. W. Kolar, "The three-phase common-mode inductor: Modeling and design issues," IEEE Transactions on Industrial Electronics, vol. 58, no. 8, pp. 3264-3274, 2010.

[21] C. Dominguez-Palacios, J. Bernal, and M. M. Prats, "Characterization of common mode chokes at high frequencies with simple measurements," IEEE Transactions on Power Electronics, vol. 33, no. 5, pp. 3975-3987, 2017.

[22] M. L. Heldwein, T. Nussbaumer, and J. W. Kolar, "Common mode modelling and filter design for a threephase buck-type pulse width modulated rectifier system," IET Power Electronics, vol. 3, no. 2, pp. 209-218, 2010.

[23] T. Nussbaumer, M. L. Heldwein, and J. W. Kolar, "Common mode EMC input filter design for a three-phase buck-type PWM rectifier system," in Proceedings of the 21st Annual IEEE Applied Power Electronics Conference and Exposition, Dallas, TX, 2006, pp. 1-7.

[24] S. Kimura, M. Takayama, T. Tajima, N. Shiba, K. Iri, and H. Aoki, "Common mode choke coil," U.S. Patent 6348 850B1, February 19, 2002.

[25] S. Raeker, "Differences between sectional and bifilar common mode chokes," 2019; https://forum.digikey.com/t/di fferences-between-sectional-and-bifilar-common-modechokes $/ 3163$.

[26] Q. Yu, T. W. Holmes, and K. Naishadham, "RF equivalent circuit modeling of ferrite-core inductors and characterization of core materials," IEEE Transactions on Electromagnetic Compatibility, vol. 44, no. 1, pp. 258-262, 2002.

[27] G. Lei, P. Dong, S. Mo, S. Yang, Z. Wu, and S. Gai, "Calculation of full permeability tensor for fractured anisotropic media," Journal of Petroleum Exploration and Production Technology, vol. 5, no. 2, pp. 167-176, 2015.

[28] S. M. Abbas, A. K. Dixit, R. Chatterjee, and T. C. Goel, "Complex permittivity, complex permeability and microwave absorption properties of ferrite-polymer composites," Journal of Magnetism and Magnetic Materials, vol. 309, no. 1, pp. 20-24, 2007.

[29] L. D. Landau, J. Bell, M. Kearsley, L. Pitaevskii, E. Lifshitz, and J. Sykes, Electrodynamics of Continuous Media (Vol. 8). Oxford, UK: Elsevier Butterworth-Heinemann, 2013.

[30] M. G. Silveirinha, "Examining the validity of KramersKronig relations for the magnetic permeability," Physical Review B, vol. 83, no. 16, article no. 165119, 2011.

[31] M. Kovacic, Z. Hanic, S. Stipetic, S. Krishnamurthy, and D. Zarko, "Analytical wideband model of a commonmode choke," IEEE Transactions on Porwer Electronics, vol. 27, no. 7, pp. 3173-3185, 2011.

[32] X. Wu, D. Xu, Z. Wen, Y. Okuma, and K. Mino, "Design, modeling, and improvement of integrated EMI filter with flexible multilayer foils," IEEE Transactions on Power Elec- 
tronics, vol. 26, no. 5, pp. 1344-1354, 2010.

[33] VACUUMSCHMELZE, "EMC products based on nanocrystalline Vitroperm," 2016; https://vacuumschmelze. de/Assets-Web/en\%20NanocrystallineVITROPERM-

Seyed Fariborz Zarei

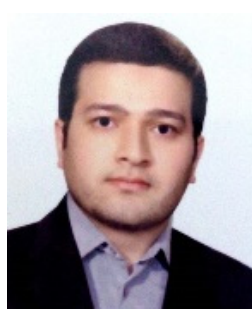

received a Ph.D. degree from the Department of Electrical Engineering, Sharif University of Technology (SUT), Tehran, Iran, in 2019. His research is focused on the application of power electronic systems in power systems. He is currently an Assistant Professor at Qom University of Technology, Qom, Iran.
EMC-Products-2016_01.pdf.

[34] VACUUMSCHMELZE, "Three-phase common mode choke (No. T60405-S6123-X225)," 2004; https://www.m ouser.com/datasheet/2/599/6123-X225-240786.pdf.

Saeed Khankalantary

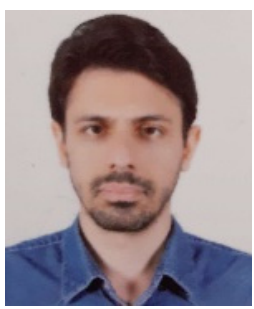

received a Ph.D. degree from the Department of Electrical Engineering, Isfahan University of Technology, Isfahan, Iran, in 2019. His research is focused on the control studies. He is currently a research assistant at Isfahan University of Technology. 